\title{
Hybrid (laparoscopy + stent) treatment of celiac trunk compression syndrome (Dunbar syndrome, median arcuate ligament syndrome (MALS))
}

\author{
Maciej Michalik ${ }^{1}$, Natalia Dowgiałto-Wnukiewicz ${ }^{1,2}$, Paweł Lech ${ }^{1}$, Kaja Majda ${ }^{1}$, Piotr Gutowski ${ }^{3}$ \\ ${ }^{1}$ Department of General and Minimally Invasive Surgery, University of Warmia and Mazury, Olsztyn, Poland \\ ${ }^{2}$ Department of Anatomy, University of Warmia and Mazury, Olsztyn, Poland \\ ${ }^{3}$ Department of Vascular Surgery, Pomeranian Medical University, Szczecin, Poland
}

Videosurgery Miniinv 2016; 11 (4): 236-239

DOI: https://doi.org/10.5114/wiitm.2016.64070

\begin{abstract}
Introduction: Celiac trunk (CT) compression syndrome caused by the median arcuate ligament (MAL) is a rarely diagnosed disease because of its nonspecific symptoms, which cause a delay in the correct diagnosis. Intestinal ischemia occurs, which causes symptoms of abdominal angina. One method of treatment for this disease is surgical release of the CT - the intersection of the MAL. Laparoscopy is the first step of the hybrid technique combined with percutaneous angioplasty and stenting of the CT.

Aim: To demonstrate the usefulness and advantages of the laparoscopic approach in the treatment of Dunbar syndrome.

Material and methods: Between 2013 and 2016 in the General and Minimally Invasive Surgery Department of the Medical Sciences Faculty of the University of Warmia and Mazury in Olsztyn, 6 laparoscopic procedures were performed because of median arcuate ligament syndrome. During the laparoscopy the MAL was cut with a harmonic scalpel. One month after laparoscopy 5 patients had Doppler percutaneous angioplasty of the CT with stent implantation in the Vascular Surgery Department in Pomeranian Medical University in Szczecin.

Results: In one case, there was a conversion of laparoscopic surgery to open due to unmanageable intraoperative bleeding. In one case, postoperative ultrasound examination of the abdominal cavity demonstrated the presence of a large hematoma in the retroperitoneal space. All patients reported relief of symptoms in the first days after the operation.

Conclusions: The hybrid method, combining laparoscopy and angioplasty, seems to be a long-term solution, which increases the comfort of the patient, brings the opportunity for normal functioning and minimizes the risk of restenosis.
\end{abstract}

Key words: laparoscopy, hybrid technique, median arcuate ligament syndrome, Dunbar syndrome, stent implantation.

\section{Introduction}

Celiac trunk (CT) compression syndrome caused by median arcuate ligament (MAL) is a rarely diagnosed disease because of its nonspecific symptoms, which cause a delay in the correct diagnosis. It was first described by J.D. Dunbar in 1965 [1, 2]. Median arcuate ligament syndrome (MALS) usually affects young people (20-40 years old), thin, often women. As a result of this condition intestinal ischemia occurs, which in $80 \%$ of people causes symptoms of abdominal angina (AA) - abdominal pain occurring after ingestion of food. However, $20 \%$ of cases are

\section{Address for correspondence}

Natalia Dowgiałło-Wnukiewicz MD, Department of General and Minimally Invasive Surgery, University of Warmia and Mazury,

2 Michała Oczapowskiego St, 10-719 Olsztyn, Poland, phone: +48 604158 786, e-mail: natalia.dowgiallo@gmail.com 
asymptomatic $[2,3]$. Nutritional disorders can result in weight loss in a relatively short time. The final diagnosis is established on the basis of imaging - Doppler ultrasound, computed angiotomography, angiography and magnetic resonance angiography $[4,5]$. One method of treatment for this disease is surgical release of the $C T$ - the intersection of the MAL $[6,7]$. Using laparoscopy can decrease the trauma and is the first step of the hybrid technique combined with percutaneous angioplasty and stenting of the CT. An excellent therapeutic effect can be achieved. Laparoscopic surgery can lead to rapid clinical improvement and resolution of symptoms, and is characterized by a small number of complications, quick convalescence and an admirable cosmetic effect. The laparoscopic surgery alone can often eliminate discomfort, while angioplasty and stent implantation are no longer necessary [7-9].

\section{Aim}

The aim of the study was to demonstrate the usefulness and advantages of laparoscopic approach in the treatment of Dunbar syndrome.

\section{Material and methods}

\section{Patients}

Between 2013 and 2016 in the General and Minimally Invasive Surgery Department of the Medical Sciences Faculty of the University of Warmia and Mazury in Olsztyn, 6 laparoscopic procedures were performed because of MALS. Among the patients there were 3 women and 3 men aged from 22 to 40 years. Symptoms of AA lasted from 8 months to 12 years. During this period one patient was treated with a diagnosis of chest pain several times at the Emergency Department, 3 people received treatment for gastric

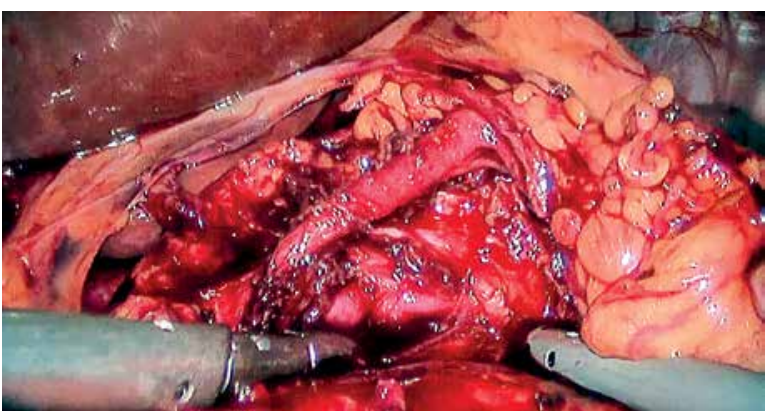

Photo 1. Celiac trunk and compressing median arcuate ligament and duodenal ulceration, and 2 patients were treated with the diagnosis of biliary colic, despite the lack of gall stones in ultrasound examination. Average body mass index (BMI) of patients was $19.25 \mathrm{~kg} / \mathrm{m}^{2}$.

The correct diagnosis was achieved as a result of Doppler ultrasound, computed angiotomography or angiography, or a combination of these tests. Other causes of abdominal pain were excluded by abdominal ultrasound, endoscopy of the upper gastrointestinal tract, test for Helicobacter pylori infection, electrocardiography and laboratory tests excluding myocardial ischemia.

\section{Surgical technique}

The patient is positioned supine on the operating table with lower extremities abducted. The table is in an anti-Trendelenburg position. Carbon dioxide is insufflated into the peritoneal cavity to a pressure of $12 \mathrm{~mm} \mathrm{Hg}$. The first $10 \mathrm{~mm}$ trocar for the camera is introduced in the midline about $12 \mathrm{~cm}$ below the xiphoid process of the sternum. After a thorough inspection of the whole peritoneal cavity under the control of the vision, the remaining trocars are inserted: $5 \mathrm{~mm}$ in the right axillary line below the costal arch (retractor of liver), $5 \mathrm{~mm}$ in the right mid-clavicular line (dissector), $10 \mathrm{~mm}$ in the left mid-clavicular line (harmonic knife) and $5 \mathrm{~mm}$ in the left anterior axillary line under the left costal arch (assist).

After inspection throughout the peritoneal cavity, the pars flaccida of the hepatogastric ligament was cut, revealing the left crus of the diaphragm. The CT and compressing ligament were exposed (Photo 1). The MAL was cut with a harmonic scalpel, which resulted in immediate filling with blood and increased diameter of the vessel (Photo 2). Then thorough hemostasis was performed. The drains were not inserted into the operating area. Patients were discharged

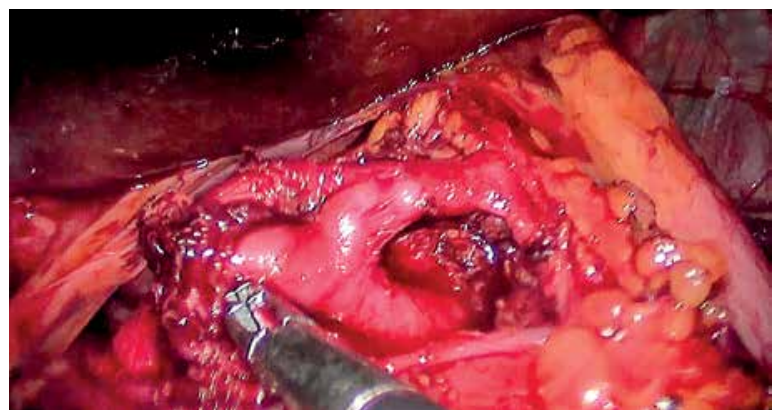

Photo 2. Celiac trunk after cutting median arcuate ligament 
on the first or second postoperative day. One patient left the clinic on the fourth postoperative day due to severe pain.

One month after laparoscopy 5 patients had Doppler percutaneous angioplasty of the CT with stent implantation in the Vascular Surgery Department in Pomeranian Medical University in Szczecin. One patient refused an additional procedure. The guidewire Terumo was inserted into the CT by the $6 \mathrm{Fr}$ introducer sheath through the right femoral or left radial artery according to the Seldinger method. Then balloon angioplasty of the CT was performed. After predilatation the bare-metal stent was implanted, adjusted to the diameter and length of the vessel. Control angiography was performed. The punctured artery was compressed for $2 \mathrm{~h}$. During the procedure $5000 \mathrm{U}$ of unfractionated heparin were administered; afterward patients have taken 75-100 mg aspirin a day. Patients were discharged on the first postoperative day.

\section{Results}

Six laparoscopic procedures were performed in the General and Minimally Invasive Surgery Department of Medical Science of University of Warmia and Mazury in Olsztyn and stent implantation was performed in 5 patients one month after in the Vascular Surgery Department in Pomeranian Medical University in Szczecin.

In one case, there was a conversion of laparoscopic surgery to open due to unmanageable intraoperative bleeding; the administration of blood components was not needed. In one case, because of persistent pain and a decrease in morphology in the postoperative period, ultrasound examination of the abdominal cavity was performed. It demonstrated the presence of a large hematoma in the retroperitoneal space at the surgical site. All patients reported relief of symptoms in the first days after the operation. The observation and control ultrasound examination as well as the supplementation of blood deficit were completed.

\section{Discussion}

Median arcuate ligament syndrome has nonspecific symptoms, which makes it difficult to diagnose and leads to a delay in the correct diagnosis. The only way to complete the diagnosis of MALS is by abnormalities on imaging studies in correlation with clinical symptoms [10]. The severity of the symptoms correlates with the degree of stenosis of the CT [11, 12]. The first symptoms appear with the reduced flow through the lumen of the CT from $50 \%$ to $75 \%$, increased severity of symptoms occurs at $75-90 \%$, and with critical stenosis, when $90 \%$ of the lumen is constricted, the full-blown form of AA is observed $[13,14]$. Abdominal organs are supplied by three major vessels extending from the aorta: the $\mathrm{CT}$, the superior mesenteric artery (SMA) and the inferior mesenteric artery (IMA). Narrowing of the CT causes gastrointestinal symptoms, while isolated narrowing of the SMA or IMA does not cause abdominal symptoms $[5,8,9,15,16]$. Only their associated constriction leads to the occurrence of AA. The respiratory phase affects the severity of symptoms. The exhalation aggravates the symptoms. The reason is the mechanism of lifting the diaphragm up, which pulls the MAL, passing in a place where the right and left crura join before branching of the CT from the aorta, compresses it and causing pain. The CT leaves at the level of Th11-L1. High branching of this short vessel of the aorta correlates with the occurrence of MALS. Nerve fibers running close to the CT may have an impact on narrowing of the flow by its direct pressure. It is also worth mentioning that the onset of Dunbar syndrome is affected by the anatomical topography (anomalies occur in 25\% of patients) and histological structure of the MAL (scar ligament tissue, in contrast to normal tissue, increases the risk of MALS) $[8,9,17,18]$.

There have been reports of using the technique of robotic surgery for intersection of the MAL [11, 14]. The hybrid method, a combination of laparoscopic patency of the $\mathrm{CT}$ and then stenting the vessel, is the best way to treat MALS, as is confirmed by the described cases. After laparoscopic surgery pain is almost immediately relieved, although on the first postoperative day it requires administration of painkillers "inadequate" for surgical trauma. The pain is probably related to the manipulation around the celiac plexus. Due to the risk of recurrence of symptoms, patients were qualified for percutaneous angioplasty and stent implantation. Stenting alone could not be performed because of technical issues. The angle of branching the compressed CT is too acute to insert the stent; moreover, the diameter of the vessel is strictured by the ligament. Because of the short time after the surgery and a small number of surgical procedures, we do not have data about 
the risk of recurrence after laparoscopic release of the CT without postoperative angioplasty. However, the combination of these two methods (laparoscopy and angioplasty) appears to be a satisfactory longterm solution $[7,13,19,20]$.

\section{Conclusions}

The hybrid method, combining laparoscopy and angioplasty, seems to be a long-term solution, which increases the comfort of the patient, brings the opportunity for normal functioning and minimizes the risk of restenosis. Laparoscopic surgery is safe and technically relatively easy, especially for surgeons familiar with procedures in the upper part of the gastrointestinal tract.

\section{Conflict of interest}

The authors declare no conflict of interest.

\section{References}

1. Arazinska A, Polguj M, Wojciechowski A, et al. Median arcuate ligament syndrome - predictor of ischemic complications? Clin Anat 2016; 29: 1025-30.

2. Mak GZ, Lucchetti AR, Drossos T, et al. Pediatric chronic abdominal pain and median arcuate ligament syndrome: a review and psychosocial comparison. Pediatr Ann 2016; 45: 257-64.

3. Harr JN, Haskins IN, Brody F. Median arcuate ligament syndrome in athletes. Surg Endosc 2016 in press.

4. Celik S, Ringe KI, Boru CE, et al. A case of pancreatic cancer with concomitant median arcuate ligament syndrome treated successfully using an allograft arterial transposition. J Surg Case Rep 2015; 2015: rjv161.

5. Akan D, Ozel A, Orhan O, et al. Doppler ultrasound diagnosis of an unusual variant of median arcuate ligament syndrome: concomitant involvement of celiac and superior mesenteric arteries. A case report. Med Ultrason 2015; 17: 557-60.

6. Lee JJ, Mills JL Sr. Chronic mesenteric ischemia from diaphrag matic compression of the celiac and superior mesenteric arteries. Ann Vasc Surg 2016; 30: 311.e5-8.

7. Rubinkiewicz M, Ramakrishnan PK, Henry BM, et al. Laparoscopic decompression as treatment for median arcuate ligament syndrome. Ann R Coll Surg Engl 2015; 97: e96-9.

8. Tembey RA, Bajaj AS, Wagle PK, et al. Real-time ultrasound: key factor in identifying celiac artery compression syndrome. Indian J Radiol Imaging 2015; 25: 202-5.

9. Baskan O, Kaya E, Gungoren FZ, et al. Compression of the celiac artery by the median arcuate ligament: multidetector computed tomography findings and characteristics. Can Assoc Radiol J 2015; 66: 272-6.

10. Columbo JA, Trus T, Nolan B, et al. Contemporary management of median arcuate ligament syndrome provides early symptom improvement. J Vasc Surg 2015; 62: 151-6.
11. You JS, Cooper M, Nishida S, et al. Treatment of median arcuate ligament syndrome via traditional and robotic techniques. Hawaii J Med Public Health 2013; 72: 279-81.

12. Joyce DD, Antiel RM, Oderich G, et al. Pediatric median arcuate ligament syndrome: surgical outcomes and quality of life. J Laparoendosc Adv Surg Tech A 2014; 24: 104-10.

13. Sultan S, Hynes N, Elsafty $N$, et al. Eight years experience in the management of median arcuate ligament syndrome by decompression, celiac ganglion sympathectomy, and selective revascularization. Vasc Endovascular Surg 2013; 47: 614-9.

14. Do MV, Smith TA, Bazan HA, et al. Laparoscopic versus robotassisted surgery for median arcuate ligament syndrome. Surg Endosc 2013; 27: 4060-6.

15. Saglam M, Sildiroglu HO, Incedayi M, et al. A variant of the median arcuate ligament syndrome: are sagittal images enough for diagnosis? Wien Klin Wochenschr 2013; 125: 220-2.

16. Jimenez JC, Harlander-Locke M, Dutson EP. Open and laparoscopic treatment of median arcuate ligament syndrome. J Vasc Surg 2012; 56: 869-73.

17. Nguyen T, Neale M, Lane R, et al. Laparoscopic management of the median arcuate ligament syndrome. ANZ J Surg 2012; 82: $265-8$

18. Gruber H, Loizides A, Peer S, et al. Ultrasound of the median arcuate ligament syndrome: a new approach to diagnosis. Med Ultrason 2012; 14: 5-9.

19. Doyle AJ, Chandra A. Chronic mesenteric ischemia in a 26-yearold man: multivessel median arcuate ligament compression syndrome. Ann Vasc Surg 2012; 26: 108.e5-9.

20. Tulloch AW, Jimenez JC, Lawrence PF, et al. Laparoscopic versus open celiac ganglionectomy in patients with median arcuate ligament syndrome. J Vasc Surg 2010; 52: 1283-9.

Received: 30.09.2016, accepted: 11.11.2016 